\title{
Covariants of binary sextics and vector-valued Siegel modular forms of genus two
}

\author{
Fabien Cléry ${ }^{1}$. Carel Faber ${ }^{2}$. \\ Gerard van der Geer $^{3}$ (i)
}

\begin{abstract}
We extend Igusa's description of the relation between invariants of binary sextics and Siegel modular forms of degree 2 to a relation between covariants and vector-valued Siegel modular forms of degree 2. We show how this relation can be used to effectively calculate the Fourier expansions of Siegel modular forms of degree 2.
\end{abstract}

Mathematics Subject Classification 10D $\cdot 11 \mathrm{~F} 11 \cdot 14 \mathrm{~L} 24 \cdot 13 \mathrm{~A} 50$

\section{Introduction}

In his 1960 papers Igusa explained the relation between the invariant theory of binary sextics and scalar-valued Siegel modular forms of degree (or genus) 2. The relation stems from the fact that the moduli space $\mathcal{M}_{2}$ of curves of genus 2 admits two descriptions. The moduli space $\mathcal{M}_{2}$ has a classical description in terms of the invariant theory

Communicated by Toby Gee.

Gerard van der Geer

G.B.M.vanderGeer@uva.nl

Fabien Cléry

cleryfabien@gmail.com

Carel Faber

C.F.Faber@uu.nl

1 Max-Planck-Institut für Mathematik, Vivatsgasse 7, 53111 Bonn, Germany

2 Mathematisch Instituut, Universiteit Utrecht, P.O. Box 80010, 3508 TA Utrecht, The Netherlands

3 Korteweg-de Vries Instituut, Universiteit van Amsterdam, Postbus 94248, 1090 GE Amsterdam, The Netherlands 
of binary sextics. But via the Torelli morphism $\mathcal{M}_{2}$ is an open part of a Shimura variety, the moduli space $\mathcal{A}_{2}$ of principally polarized abelian surfaces. Therefore we have two descriptions of natural vector bundles on our moduli space and thus two descriptions of their sections. The purpose of this paper is to extend the correspondence given by Igusa between invariants of binary sextics and scalar-valued Siegel modular forms of degree 2 to a correspondence between covariants and vector-valued Siegel modular forms of degree 2. We give a description of vector-valued Siegel modular forms of degree 2 in terms of covariants of the action of $\operatorname{SL}(2, \mathbb{C})$ on the space of binary sextics.

Since the complement of the Torelli image of $\mathcal{M}_{2}$ in $\mathcal{A}_{2}$ is the space $\mathcal{A}_{1,1}$ of decomposable abelian surfaces (products of elliptic curves) we have to analyze the orders of modular forms described by covariants along this locus. The scalar-valued modular forms $\chi_{10}$ and its square root $\chi_{5}$ that vanish with multiplicity 2 and 1 along this locus play a key role.

We shall see that the 'first' vector-valued modular form that appears on $\operatorname{Sp}(4, \mathbb{Z})$, the form $\chi_{6,8}$ of weight $(6,8)$, corresponds in some sense to the 'universal' binary sextic. The relation between covariants and modular forms allows us to use constructions from representation theory to construct vector-valued Siegel modular forms. In fact, we shall show that up to multiplication by a suitable power of $\chi_{10}$, every vector-valued Siegel modular form of degree 2 can be obtained by applying a representation-theoretic construction to the form $\chi_{6,8}$. In this sense, our form $\chi_{6,8}$ may be called the 'universal vector-valued Siegel modular form of degree 2 '. In fact, in practice we work with the form $\chi_{6,3}=\chi_{6,8} / \chi_{5}$ which is a modular form with a character.

We show that our constructions can be used to efficiently calculate the Fourier expansions of vector-valued Siegel modular forms. We illustrate this with a number of significant examples where we use these Fourier expansions to calculate eigenvalues of Hecke operators and check instances of Harder type congruences.

Similar ideas will be worked out for the case of genus 3 in a forthcoming paper.

\section{Siegel modular forms}

The Siegel modular group $\Gamma_{g}=\operatorname{Sp}(2 g, \mathbb{Z})$ of degree (or genus) $g$ acts on the Siegel upper half-space

$$
\mathfrak{H}_{g}=\left\{\tau \in \operatorname{Mat}(g \times g, \mathbb{C}): \tau^{t}=\tau, \operatorname{Im}(\tau)>0\right\}
$$

of degree $g$ by

$$
\tau \mapsto \gamma \cdot \tau=(a \tau+b)(c \tau+d)^{-1} \quad \text { for } \gamma=\left(\begin{array}{ll}
a & b \\
c & d
\end{array}\right) \in \Gamma_{g}
$$

A scalar-valued Siegel modular form of degree $g>1$ and weight $k$ is a holomorphic function $f: \mathfrak{H}_{g} \rightarrow \mathbb{C}$ satisfying

$$
f(\gamma \cdot \tau)=\operatorname{det}(c \tau+d)^{k} f(\tau)
$$


for all $\gamma \in \Gamma_{g}$. (For $g=1$ we also need a growth condition at infinity.) If $W$ is a finitedimensional complex vector space and $\rho: \operatorname{GL}(g, \mathbb{C}) \rightarrow \mathrm{GL}(W)$ a representation, then a vector-valued Siegel modular form of degree $g>1$ and weight $\rho$ is a holomorphic map $f: \mathfrak{H}_{g} \rightarrow W$ such that

$$
f(\gamma \cdot \tau)=\rho(c \tau+d) f(\tau)
$$

for all $\gamma \in \Gamma_{g}$. Siegel modular forms can be interpreted as sections of vector bundles. The moduli space $\mathcal{A}_{g}(\mathbb{C})=\Gamma_{g} \backslash \mathfrak{H}_{g}$ of complex principally polarized abelian varieties of dimension $g$ carries an orbifold vector bundle of rank $g$, the Hodge bundle $\mathbb{E}$, defined by the factor of automorphy given by

$$
j(\gamma, \tau)=(c \tau+d) .
$$

Its determinant $L=\operatorname{det}(\mathbb{E})$ defines a line bundle. A scalar-valued Siegel modular form of degree $g$ and weight $k$ can be seen as a section of $L^{\otimes k}$. A vector-valued Siegel modular form of weight $\rho$ can be viewed as a section of the vector bundle $\mathbb{E}_{\rho}$ that is defined by the factor of automorphy $j(\gamma, \tau)=\rho(c \tau+d)$. The vector bundle $\mathbb{E}$ and the bundles $\mathbb{E}_{\rho}$ extend in a canonical way to toroidal compactifications of $\mathcal{A}_{g}$ and the Koecher principle says that their sections do so too.

We are interested in the case $g=2$. An irreducible representation of GL(2, $\mathbb{C})$ is of the form $\operatorname{Sym}^{j}(V) \otimes \operatorname{det}(V)^{\otimes k}$ for $(j, k) \in \mathbb{Z}_{\geq 0} \times \mathbb{Z}$ with $V$ the standard representation of $\operatorname{GL}(2, \mathbb{C})$. We denote the corresponding vector space of Siegel modular forms by $M_{j, k}\left(\Gamma_{2}\right)$ or simply by $M_{j, k}$. It contains a subspace of cusp forms denoted by $S_{j, k}\left(\Gamma_{2}\right)$ or simply by $S_{j, k}$. The scalar-valued modular forms correspond to the case where $j=0$.

The scalar-valued modular forms of degree 2 form a ring $R=\oplus_{k} M_{0, k}\left(\Gamma_{2}\right)$. Igusa showed in [14] that $R$ is generated by modular forms $E_{4}, E_{6}, \chi_{10}, \chi_{12}$ and $\chi_{35}$ of weight $4,6,10,12$ and 35 . The subring $R^{\mathrm{ev}}$ of modular forms of even weight is the pure polynomial ring generated by $E_{4}, E_{6}, \chi_{10}$ and $\chi_{12}$, and the form $\chi_{35}$ satisfies a quadratic equation over $R^{\mathrm{ev}}$ expressing $\chi_{35}^{2}$ as a polynomial in $E_{4}, E_{6}, \chi_{10}$ and $\chi_{12}$.

The bi-graded $R$-module $M=\oplus_{j, k} M_{j, k}$ can also be made into a ring by using the canonical projections $\operatorname{Sym}^{j_{1}}(V) \otimes \operatorname{Sym}^{j_{2}}(V) \rightarrow \operatorname{Sym}^{j_{1}+j_{2}}(V)$ defined by multiplying homogeneous polynomials of degree $j_{1}$ and $j_{2}$ in two variables to define the multiplication.

The ring $M$ of vector-valued modular forms is not finitely generated as shown by Grundh, see [9, p. 234].

\section{Invariants and covariants of binary sextics}

Let $V$ be a 2-dimensional vector space (over $\mathbb{C}$ ), generated by elements $x_{1}$ and $x_{2}$. We will denote by $\operatorname{Sym}^{6}(V)$ the space of binary sextics, where we write a binary sextic as

$$
f=\sum_{i=0}^{6} a_{i}\left(\begin{array}{l}
6 \\
i
\end{array}\right) x_{1}^{6-i} x_{2}^{i}
$$


The group $\mathrm{GL}(2, \mathbb{C})$ acts on $V$ via $\left(x_{1}, x_{2}\right) \mapsto\left(a x_{1}+b x_{2}, c x_{1}+d x_{2}\right)$ for $(a, b ; c, d) \in \mathrm{GL}(2, \mathbb{C})$. This induces an action of $\operatorname{GL}(2, \mathbb{C})$ on $\operatorname{Sym}^{6}(V)$, and similarly actions of $\operatorname{PGL}(2, \mathbb{C})$ and $\operatorname{SL}(2, \mathbb{C})$ on $\mathcal{X}=\mathbb{P}\left(\operatorname{Sym}^{6}(V)\right)$. We take $\left(a_{0}: a_{1}\right.$ : $\left.\ldots: a_{6}\right)$ as coordinates on the projectivized space $\mathcal{X}$.

The space $\mathcal{X}$ carries the natural ample line bundle $\mathcal{L}=\mathcal{O}_{\mathbb{P}\left(\operatorname{Sym}^{6}(V)\right)}(1)$. On $\mathcal{L}$ we have an action of the group $\operatorname{SL}(2, \mathbb{C})$ that is compatible with the action on the projectivized space $\mathcal{X}$ of binary sextics, see [21]. We can retrieve $\mathcal{X}$ as the projective scheme $\operatorname{Proj}\left(\oplus_{n} \mathrm{H}^{0}\left(\mathcal{X}, \mathcal{L}^{n}\right)\right)$.

The ring of invariants is defined as the graded ring

$$
I=\oplus_{n \geq 0} \mathrm{H}^{0}\left(\mathcal{X}, \mathcal{L}^{n}\right)^{\mathrm{SL}(2, \mathbb{C})} .
$$

Thus an invariant can be seen as a polynomial in the coefficients $a_{i}$ of $f$ that is invariant under the action of $\operatorname{SL}(2, \mathbb{C})$. The ring of these invariants was determined in the 19 th century by work of Clebsch and Bolza [3,5]. It is generated by elements $A, B, C, D$ and $E$ of degrees $6,12,18,30$ and 45 in the roots of the binary sextic. We normalize their degree by taking the degree in the roots divided by 3 and this is then the degree in the $a_{i}$. The invariant $D$ is the discriminant and $E$ satisfies a quadratic equation expressing $E^{2}$ in the even invariants.

Given the representation of $\operatorname{GL}(2, \mathbb{C})$ on $V$ and on $\operatorname{Sym}^{6}(V)$ we look at equivariant inclusions of $\mathrm{GL}(2, \mathbb{C})$-representations

$$
\iota_{A}: A \longrightarrow \operatorname{Sym}^{d}\left(\operatorname{Sym}^{6}(V)\right),
$$

with $A$ a GL(2, $\mathbb{C})$-module, or equivalently at equivariant inclusions

$$
\varphi: \mathbb{C} \longrightarrow \operatorname{Sym}^{d}\left(\operatorname{Sym}^{6}(V)\right) \otimes A^{\vee} .
$$

The image $\varphi(1)$ is denoted $\Phi=\Phi(\varphi)$ and called a covariant.

If $A=A[\lambda]$ is the irreducible representation of $\operatorname{GL}(2, \mathbb{C})$ of highest weight $\lambda=$ $\left(\lambda_{1}, \lambda_{2}\right)$ with $\lambda_{1} \geq \lambda_{2}$ then the covariant $\Phi$ can be viewed as a form of degree $d$ in the variables $a_{0}, \ldots, a_{6}$, of degree $\lambda_{1}-\lambda_{2}$ in $x_{1}$ and $x_{2}$.

Example 3.1 The tautological $f$. As a first example we consider the (tautological) case where $d=1$ and $A=\operatorname{Sym}^{6}(V)$ and we take $\iota_{A}=\operatorname{id}_{\operatorname{Sym}^{6}(V)}$. Then the covariant $\Phi=\varphi(1)$ can be viewed as the universal binary sextic $f=\sum_{J} a_{J} x^{J}$ given by (1).

Example 3.2 As a second example we look at $d=2$. We have the isotypical decomposition

$$
\operatorname{Sym}^{2}\left(\operatorname{Sym}^{6}(V)\right)=A[12,0]+A[10,2]+A[8,4]+A[6,6] .
$$

We can view the covariant associated to $A[12,0]$ as the square of the tautological $f$ given by (1), while the covariant associated to $A[10,2]$ is the Hessian of the polynomial $f$ given by $f_{x_{1} x_{1}} f_{x_{2} x_{2}}-f_{x_{1} x_{2}}^{2}$ or equivalently (up to a scalar) by

$$
\left(a_{0} a_{2}-a_{1}^{2}\right) x_{1}^{8}+4\left(a_{0} a_{3}-a_{1} a_{2}\right) x_{1}^{7} x_{2}+\cdots+\left(a_{4} a_{6}-a_{5}^{2}\right) x_{2}^{8} .
$$


The covariant corresponding to $A[8,4]$ is given by

$$
\begin{aligned}
& \left(a_{0} a_{4}-4 a_{1} a_{3}+3 a_{2}^{2}\right) x_{1}^{4}+\left(2 a_{0} a_{5}-6 a_{1} a_{4}+4 a_{2} a_{3}\right) x_{1}^{3} x_{2} \\
& \quad+\left(a_{0} a_{6}-9 a_{2} a_{4}+8 a_{3}^{2}\right) x_{1}^{2} x_{2}^{2} \\
& \quad+\left(2 a_{1} a_{6}-6 a_{2} a_{5}+4 a_{3} a_{4}\right) x_{1} x_{2}^{3}+\left(a_{2} a_{6}-4 a_{3} a_{5}+3 a_{4}^{2}\right) x_{2}^{4} .
\end{aligned}
$$

Moreover, the covariant associated to $A[6,6]$ is an invariant equal to $a_{0} a_{6}-6 a_{1} a_{5}+$ $15 a_{2} a_{4}-10 a_{3}^{2}$ and coincides (up to a multiplicative scalar) with the invariant $A \in I$.

The covariants of the action of $\operatorname{SL}(2, \mathbb{C})$ form a graded ring $C$. This ring can be identified with the ring of invariants

$$
\mathbb{C}\left[V_{1} \oplus V_{6}\right]^{\mathrm{SL}(2, \mathbb{C})},
$$

where we write $V_{i}=\operatorname{Sym}^{i}(V)$, see [23, p. 55]. It was shown in the 19 th century by Clebsch and others that the ring $C$ is generated by 26 elements ( 5 invariants and 21 other covariants) [5, p. 296]. Covariants corresponding to an irreducible representation $A[\lambda]$ with $\lambda_{1}+\lambda_{2}=6 d$ can be calculated by the so-called symbolic method. We refer to [10, p. 156], [4] and [22, p. 214].

\section{Covariants and Siegel modular forms}

The moduli space of curves of genus 2 admits two different descriptions. First, using the Torelli morphism $t$ we can view $\mathcal{M}_{2}$ as an open subspace of the moduli space $\mathcal{A}_{2}$ of principally polarized abelian surfaces. The complement of the image $t\left(\mathcal{M}_{2}\right)$ in $\mathcal{A}_{2}$ is the divisor $\mathcal{A}_{1,1}$ of products of elliptic curves. Over the complex numbers we can view $\mathcal{M}_{2}(\mathbb{C})$ as an open suborbifold of the orbifold $\mathcal{A}_{2}(\mathbb{C})=\Gamma_{2} \backslash \mathfrak{H}_{2}$.

The second description of $\mathcal{M}_{2}$ is as the GIT quotient associated to the action of $\mathrm{GL}(2, \mathbb{C})$ on the space $X=\operatorname{Sym}^{6}(V)$ of binary sextics. A binary sextic $f\left(x_{1}, x_{2}\right)$ with non-vanishing discriminant determines a curve $C$ of genus 2 with affine equation $y^{2}=\tilde{f}(x)$ with $\tilde{f}(x)=f(x, 1)$. Moreover, the curve $C$ comes with a basis of the space of differentials given by $x d x / y$ and $d x / y$.

We let the group $\operatorname{GL}(2, \mathbb{C})$ act on the pairs $(x, y)$ by

$$
x \mapsto(a x+b) /(c x+d), \quad y \mapsto y /(c x+d)^{3} .
$$

This gives an action $d x \mapsto(a d-b c) d x /(c x+d)^{2}$ and hence an action by $\operatorname{det}(A) A$ on our basis $x d x / y, d x / y$.

Using this we can identify the moduli space $\mathcal{M}_{2}$ with the algebraic stack $[Y / \mathrm{GL}(2, \mathbb{C})]$ with $Y$ the algebraic stack of curves with a framed Hodge bundle: the objects of $Y$ are pairs $(\pi, \alpha)$ with $\pi: C \rightarrow S$ a curve of genus 2 and an isomorphism $\alpha: \mathcal{O}_{S}^{\oplus 2} \stackrel{\sim}{\longrightarrow} \pi_{*} \omega_{\pi}$. The group $\operatorname{GL}(2, \mathbb{C})$ acts via $(\pi, \alpha) \mapsto A \cdot(\pi, \alpha)=$ $(\pi, \operatorname{det}(A) A \circ \alpha)$. We thus have (see [24])

$$
q:[Y / \mathrm{GL}(2, \mathbb{C})] \stackrel{\sim}{\longrightarrow} \mathcal{M}_{2} .
$$


Let $X^{0} \subset X$ be the complement of the discriminant locus. We have a natural identification of $Y$ with $X^{0}$ such that the above action of GL(2, $\left.\mathbb{C}\right)$ on $Y$ corresponds to the action of $\operatorname{GL}(2, \mathbb{C})$ on $X$ given by $A \circ f=f\left(a x_{1}+b x_{2}, c x_{1}+d x_{2}\right)$. This implies the following corollary.

Corollary 4.1 The pullback of the Hodge bundle $\mathbb{E}$ on $\mathcal{M}_{2}$ to $X^{0} \cong Y$ is the $\mathrm{GL}(2, \mathbb{C})$ equivariant bundle $V \otimes \operatorname{det}(V)$.

This can be extended to include the locus of binary sextics with at least five distinct zeros. We write a binary sextic with five zeros as $(x-\alpha)^{2} f_{4}$ with $f_{4}$ a binary quartic with non-zero discriminant and $f_{4}(\alpha) \neq 0$. To this we associate the nodal curve $C$ which is obtained by associating to $f_{4}$ the genus 1 curve $C_{1}$ given by $w^{2}=\tilde{f}_{4}$ with $\tilde{f}_{4}=f_{4}(x, 1)$ and identifying the two points of $C_{1}$ lying over $x=\alpha$. It comes with two differential forms $d x / w$ and $d x /(x-\alpha) w$; the latter form has poles with opposite residues in the two points of $C_{1}$ lying over $x=\alpha$. The connection with the case of sextics with non-vanishing discriminant is given by setting $y=w(x-\alpha)$. In this way we can associate to a binary sextic with at least five zeros a nodal curve with a frame of the Hodge bundle. The group $\operatorname{GL}(2, \mathbb{C})$ acts by

$$
x \mapsto(a x+b) /(c x+d), \quad w \mapsto w /(c x+d)^{2} .
$$

The identification $[Y / \mathrm{GL}(2, \mathbb{C})] \cong \mathcal{M}_{2}$ can be extended if we include for $Y$ the case of irreducible nodal curves of arithmetic genus 2 with one node and replace $\mathcal{M}_{2}$ by $\overline{\mathcal{M}}_{2}-\Delta_{1}$ with $\Delta_{1}$ the locus of reducible curves. The conclusion of Corollary 4.1 is still valid. In a similar way it can be extended to the case of binary sextics all of whose zeros have multiplicity $\leq 2$. We denote by $X^{s}$ the open subset of $X$ of stable sextics, that is, binary sextics none of whose zeros have multiplicity $\geq 3$.

A scalar-valued Siegel modular form $F$ of weight $k$ on $\Gamma_{2}$ is by definition a section of the line bundle $L^{k}$ with $L=\operatorname{det}(\mathbb{E})$ on $\tilde{\mathcal{A}}_{2}$, the standard toroidal compactification (equal to $\overline{\mathcal{M}}_{2}$ ), and its pullback to $X^{s}$ will give rise to an invariant section of $\operatorname{det}(V)^{3 k}$ on $X^{s}$, that is, an invariant $i_{F}$, hence an invariant on all of $X$. This gives a map from the ring of scalar-valued Siegel modular forms $R$ to the ring of invariants

$$
i: R \longrightarrow I
$$

Igusa defined this injective map in a slightly different way in [16]. By Igusa's map we can view $I$ as a ring of meromorphic Siegel modular forms. The generators $E_{4}, E_{6}, \chi_{10}, \chi_{12}$ and $\chi_{35}$ of $R$ correspond to non-zero multiples of $B, A B-$ $3 C, D, A D$ and $D^{2} E$ (see [16, p. 848]). We see that every invariant defines a meromorphic modular form $F$ such that $\chi_{10}^{m} F$ is holomorphic for an appropriate power $m$. In other words we have inclusions

$$
R \subset I \subset R_{\chi_{10}}
$$

where $R_{\chi_{10}}$ is obtained by inverting $\chi_{10}$. Moreover, the ideal of $R$ of cusp forms maps to $(D)$, the ideal generated by the discriminant (cf. [16, p. 845]). 
The relation between invariants and scalar-valued Siegel modular forms can be extended to a relation between covariants and vector-valued Siegel modular forms. A section $F$ of $\operatorname{Sym}^{j}(\mathbb{E}) \otimes \operatorname{det}(\mathbb{E})^{k}$ will by pullback under $q$ give rise to a section of $\operatorname{Sym}^{j}(V) \otimes \operatorname{det}(V)^{j+3 k}$, hence to a covariant of the action of $\operatorname{SL}(2, \mathbb{C})$ on $\operatorname{Sym}^{6}(V)$. We get inclusions similar to those above

$$
M \subset C \subset M_{\chi_{10}}
$$

with $M_{\chi_{10}}=M \otimes_{R} R_{\chi_{10}}$ fitting into a commutative diagram

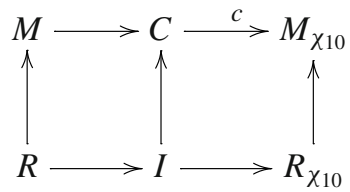

Recall that the ring $M$ of vector-valued modular forms is not finitely generated, but the ring $C$ of covariants is. Any covariant defines a meromorphic Siegel modular form the polar locus of which is either empty or the divisor $\mathcal{A}_{1,1}$. A covariant, that is, an equivariant section of $\operatorname{Sym}^{p}(V) \otimes \operatorname{det}(V)^{q}$ with $\lambda=(p+q, q)$, defines a section $F$ of

$$
\operatorname{Sym}^{p}(\mathbb{E}) \otimes \operatorname{det} \mathbb{E}^{(q-p) / 3}=\mathbb{E}_{\lambda} \otimes \operatorname{det} \mathbb{E}^{-2 d}
$$

on $\mathcal{A}_{2}-\mathcal{A}_{1,1}$. If $F$ extends to a holomorphic section of some $\operatorname{Sym}^{j}(\mathbb{E}) \otimes \operatorname{det}(\mathbb{E})^{k}$ on $\mathcal{A}_{2}$, then it extends as a section of that bundle to all of $\tilde{\mathcal{A}}_{2}$ by the Koecher principle. If the section $F$ has order $-v(F)$ along the divisor $\mathcal{A}_{1,1}$ then multiplying $F$ with $\chi_{10}^{\lceil v(F) / 2\rceil}$ makes it into a holomorphic section. Similarly, multiplication with $\chi_{5}^{v(F)}$ makes it into a holomorphic modular form with character of weight $(p,(q-p) / 3+5 v(F))$, see the next section. In particular, we can apply this to the universal binary sextic $f$ given in Example 3.1. It thus defines a meromorphic Siegel modular form of weight $(6,-2)$.

\section{The forms $\chi_{10}$ and $\chi_{6,8}$}

In this paper two modular forms (and two closely related ones) will play a central role. The modular forms are $\chi_{10}$ and $\chi_{6,8}$ and the related ones are $\chi_{5}$ and $\chi_{6,3}$. Up to a normalization the modular form $\chi_{5}$ is defined as the product of the ten even theta constants and $\chi_{10}=\chi_{5}^{2}$ is its square. The form $\chi_{5}$ can be seen as a cusp form of weight 5 on the principal congruence subgroup $\Gamma_{2}[2]$ of level 2 which is invariant under the alternating group $\mathfrak{A}_{6} \subset \mathfrak{S}_{6}=\operatorname{Sp}(4, \mathbb{Z} / 2 \mathbb{Z})$; alternatively it can be seen as a modular form on $\Gamma_{2}$ with character $\epsilon$. (The abelianization of $\Gamma_{2}$ is $\mathbb{Z} / 2 \mathbb{Z}$, see [19, Satz 3], [20].) The Fourier expansion of $\chi_{5}$ follows from its definition and starts with

$$
\begin{aligned}
\chi_{5}(\tau)= & (1 / R-R) Q_{1} Q_{2}+\left(-1 / R^{3}-9 / R+9 R+R^{3}\right)\left(Q_{1}^{3} Q_{2}+Q_{1} Q_{2}^{3}\right) \\
& +\left(-9 / R^{5}+93 / R^{3}-90 / R+90 R-93 R^{3}+9 R^{5}\right) Q_{1}^{3} Q_{2}^{3}+\cdots,
\end{aligned}
$$


where we write $\tau \in \mathfrak{H}_{2}$ as

$$
\tau=\left(\begin{array}{cc}
\tau_{1} & \tau_{12} \\
\tau_{12} & \tau_{2}
\end{array}\right)
$$

and use $Q_{1}=e^{\pi i \tau_{1}}, Q_{2}=e^{\pi i \tau_{2}}$ and $R=e^{\pi i \tau_{12}}$. The Fourier expansion of $\chi_{10}$ thus starts with

$$
\begin{aligned}
\chi_{10}(\tau)= & (1 / r-2+r) q_{1} q_{2}-\left(2 / r^{2}+16 / r-36+16 r+2 r^{2}\right)\left(q_{1}^{2} q_{2}+q_{1} q_{2}^{2}\right) \\
& +\left(1 / r^{3}+36 / r^{2}+99 / r-272+99 r+36 r^{2}+r^{3}\right)\left(q_{1}^{3} q_{2}+q_{1} q_{2}^{3}\right) \\
& +\left(-16 / r^{3}+240 / r^{2}-240 / r+32-240 r+240 r^{2}-16 r^{3}\right) q_{1}^{2} q_{2}^{2} \\
& +\cdots,
\end{aligned}
$$

where $q_{1}=e^{2 \pi i \tau_{1}}, q_{2}=e^{2 \pi i \tau_{2}}$ and $r=e^{2 \pi i \tau_{12}}$.

We will be interested in the expansion of $\chi_{5}$ and $\chi_{10}$ along the locus $\mathfrak{H}_{1} \times \mathfrak{H}_{1} \subset \mathfrak{H}_{2}$ given by $\tau_{12}=0$. Note that the pullback to $\mathcal{A}_{1} \times \mathcal{A}_{1}$ of the normal bundle along $\mathcal{A}_{1,1}$ is the dual of the product $\mathbb{E} \otimes \mathbb{E}$ of the (pullbacks of the) Hodge bundles of the two factors, see [6, p. 23].

We need the concept of a quasi-modular form and refer to [18] and to [25]. For even $k \geq 2$, we will denote the Eisenstein series of weight $k$ on $\operatorname{SL}(2, \mathbb{Z})$ by $e_{k}$. Its Fourier expansion is given by

$$
e_{k}(\tau)=1-\frac{2 k}{B_{k}} \sum_{n \geqslant 1} \sigma_{k-1}(n) q^{n}
$$

with $B_{k}$ the $k$ th Bernoulli number, $\sigma_{r}(n)=\sum_{d \mid n} d^{r}$ and $q=e^{2 \pi i \tau}$. For a subgroup $\Gamma$ of finite index of the full modular group $\operatorname{SL}(2, \mathbb{Z})$ we denote by $M_{*}(\Gamma)=\oplus_{k} M_{k}(\Gamma)$ the graded ring of modular forms and by $\widetilde{M}_{*}(\Gamma)=\oplus_{k} \widetilde{M}_{k}(\Gamma)$ the graded ring of quasi-modular forms on $\Gamma$. We have a differential operator

$$
D=\frac{1}{2 \pi i} \frac{d}{d \tau}=q \frac{d}{d q}
$$

that sends quasi-modular forms to quasi-modular forms. We refer to [18] for the following facts.

Lemma 5.1 We have $\widetilde{M}_{*}(\Gamma)=M_{*}(\Gamma) \otimes \mathbb{C}\left[e_{2}\right]$. Furthermore, for even $k>0$ we have

$$
\widetilde{M}_{k}(\Gamma)=\oplus_{0 \leqslant i \leqslant k / 2} D^{i} M_{k-2 i}(\Gamma) \oplus\left\langle D^{k / 2-1} e_{2}\right\rangle .
$$

We develop $\chi_{10}$ as a Taylor series in the normal direction of $\mathfrak{H}_{1} \times \mathfrak{H}_{1}$ inside $\mathfrak{H}_{2}$ with coordinate $t=2 \pi i \tau_{12}$

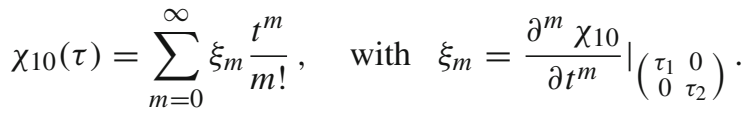


Since sections of the Hodge bundle on $\tilde{\mathcal{A}}_{1}$ are modular forms and since we know that the operator $D$ sends the ring of quasi-modular forms on $\operatorname{SL}(2, \mathbb{Z})$ to itself, it follows that the terms in the Taylor series of $\chi_{10}$ on $\mathfrak{H}_{2}$ along $\mathfrak{H}_{1} \times \mathfrak{H}_{1}$ are quasi-modular forms. Using the definition in terms of even theta constants we can calculate the expansions of $\chi_{10}$ and $\chi_{5}$.

Lemma 5.2 We have $\xi_{m}=0$ for every odd $m$. The first three non-zero coefficients of $\chi_{10}=\sum_{m=0}^{\infty} \xi_{m} t^{m} / m$ ! are

$$
\xi_{2}=2 \Delta \otimes \Delta, \quad \xi_{4}=2 \Delta e_{2} \otimes \Delta e_{2},
$$

and

$$
\xi_{6}=\frac{1}{24}\left(-7 \Delta e_{4} \otimes \Delta e_{4}+65 \Delta e_{2}^{2} \otimes \Delta e_{2}^{2}-5\left(\Delta e_{4} \otimes \Delta e_{2}^{2}+\Delta e_{2}^{2} \otimes \Delta e_{4}\right)\right) .
$$

Here we use the shorthand $f \otimes g$ instead of $f\left(\tau_{1}\right) \otimes g\left(\tau_{2}\right)$.

Similarly, for the development of $\chi_{5}$ as a Taylor series in the normal direction we need the square root of $\Delta$ which is the modular form

$$
\delta=q^{1 / 2} \prod_{n=1}^{\infty}\left(1-q^{n}\right)^{12}
$$

of weight 6 on the congruence subgroup $\Gamma_{1}(2)$. If we use $s=\pi i \tau_{12}$ as the normal coordinate then we find the development

$$
\chi_{5}(\tau)=-2 \delta \otimes \delta s-\frac{1}{3} \delta e_{2} \otimes \delta e_{2} s^{3}+\cdots
$$

The second form that plays a central role is the form $\chi_{6,8}$ (and its relative $\chi_{6,3}$ ). We defined this vector-valued Siegel modular form of weight $(6,3)$ with character $\epsilon$ on $\Gamma_{2}$ in the paper [7, Example 16.1] using the gradients of the six odd theta series $\vartheta_{m_{i}}(\tau, z)(i=1, \ldots, 6)$ with characteristics in degree 2 :

$$
\chi_{6,3}=c \cdot \operatorname{Sym}^{6}\left(G_{1}, \ldots, G_{6}\right) \quad \text { with } \quad G_{i}(\tau)=\left(\begin{array}{c}
\partial \vartheta_{m_{i}} / \partial z_{1} \\
\partial \vartheta_{m_{i}} / \partial z_{2}
\end{array}\right)(\tau,(0,0))
$$

here the constant $c \in \mathbb{C}^{*}$ is chosen such that the Fourier expansion of $\chi_{6,3}$ starts with

$$
\chi_{6,3}(\tau)=\left(\begin{array}{c}
0 \\
0 \\
\left(R-R^{-1}\right) \\
\left(2 R+2 R^{-1}\right) \\
\left(R-R^{-1}\right) \\
0 \\
0
\end{array}\right) Q_{1} Q_{2}+\cdots,
$$

where $Q_{1}=e^{i \pi \tau_{1}}, Q_{2}=e^{i \pi \tau_{2}}$ and $R=e^{i \pi \tau_{12}}$. The form $\chi_{6,3}$ is a modular form of weight $(6,3)$ with character $\epsilon$ on $\Gamma_{2}$. (For the coordinates chosen on $\operatorname{Sym}^{6}$ we 
refer to the beginning of Section 3.) Alternatively, it can be viewed as a form on the level 2 principal congruence subgroup $\Gamma_{2}$ [2] that is invariant under the action of the alternating group $\mathfrak{A}_{6}$. We define $\chi_{6,8} \in S_{6,8}\left(\Gamma_{2}\right)$ as the product $\chi_{6,3} \chi_{5}$.

Using the definition with the gradients we find the Taylor expansion of $\chi_{6,3}$ in the normal direction along $\mathfrak{H}_{1} \times \mathfrak{H}_{1}$ with $s=\pi i \tau_{12}$ as coordinate:

$$
\chi_{6,3}(\tau)=\left(\begin{array}{c}
0 \\
0 \\
0 \\
4 \delta \otimes \delta \\
0 \\
0 \\
0
\end{array}\right)+\left(\begin{array}{c}
0 \\
0 \\
2 e_{2} \delta \otimes \delta \\
0 \\
2 \delta \otimes e_{2} \delta \\
0 \\
0
\end{array}\right) s+\left(\begin{array}{c}
0 \\
\frac{2}{3}\left(e_{2}^{2}-e_{4}\right) \delta \otimes \delta \\
0 \\
4 e_{2} \delta \otimes e_{2} \delta \\
0 \\
\frac{2}{3} \delta \otimes\left(e_{2}^{2}-e_{4}\right) \delta \\
0
\end{array}\right) \frac{s^{2}}{2 !}+\cdots .
$$

This gives then the Taylor development of $\chi_{6,8}$ in the normal direction with coordinate $t=2 \pi i \tau_{12}$ :

$$
\chi_{6,8}(\tau)=-\left(\begin{array}{c}
0 \\
0 \\
0 \\
4 \Delta \otimes \Delta \\
0 \\
0 \\
0
\end{array}\right) t-\left(\begin{array}{c}
0 \\
0 \\
e_{2} \Delta \otimes \Delta \\
0 \\
\Delta \otimes e_{2} \Delta \\
0 \\
0
\end{array}\right) t^{2}-\left(\begin{array}{c}
0 \\
2\left(e_{2}^{2}-e_{4}\right) \Delta \otimes \Delta \\
0 \\
16 e_{2} \Delta \otimes e_{2} \Delta \\
0 \\
2 \Delta \otimes\left(e_{2}^{2}-e_{4}\right) \Delta \\
0
\end{array}\right) \frac{t^{3}}{3}+\cdots
$$

The modular form $\chi_{6,8}$ was first given by Ibukiyama using theta series with pluriharmonic coefficients, see [12]. It is the first vector-valued (non scalar-valued) modular form if one orders them according to Deligne weight $(=j+2 k-3)$.

\section{Modular forms associated to covariants}

Recall from Sect. 4 the inclusions

$$
M \longrightarrow C \stackrel{c}{\longrightarrow} M_{\chi_{10}}
$$

We wish to describe the map $c$ explicitly. For this we consider the meromorphic modular form $\chi_{6,3} / \chi_{5}$ of weight $(6,-2)$ (without character). It is holomorphic on $\widetilde{\mathcal{A}}_{2}-\mathcal{A}_{1,1}$, hence defines by Corollary 4.1 under pullback a covariant, a section of $\operatorname{Sym}^{6}(V)$. Up to a non-zero multiplicative scalar $r$ we thus find the tautological $f$ (see Example 3.1), hence

$$
r c(f)=\frac{\chi_{6,3}}{\chi_{5}}
$$

We will discard the factor $r$ and assume that $r=1$ by normalizing $f$ appropriately. But every covariant can be constructed from $f$. In fact, let $h$ be a covariant associated to the irreducible representation $A[p+q, q]$ in $\operatorname{Sym}^{d}\left(\operatorname{Sym}^{6}(V)\right)$. It can be viewed as a form of degree $d$ in $a_{0}, \ldots, a_{6}$, the coefficients of $f$, and of degree $p$ in the coordinates $x_{1}$ and $x_{2}$, or simply as a vector of length $p+1$ with entries which are polynomials of degree $d$ in $a_{0}, \ldots, a_{6}$. 
Now write the Fourier expansion of $\chi_{6,3}$ as a vector $\left(\alpha_{0}, 6 \alpha_{1}, 15 \alpha_{2}, 20 \alpha_{3}, \ldots, \alpha_{6}\right)^{t}$, or more symbolically as

$$
\chi_{6,3}=\sum_{i=0}^{6}\left(\begin{array}{l}
6 \\
i
\end{array}\right) \alpha_{i} X^{6-i} Y^{i}
$$

where $\alpha_{i}$ is a Fourier series in $Q_{1}, Q_{2}$ and $R$ and $R^{-1}$, and the $X^{6-i} Y^{i}$ indicate the coordinate places. Define a map

$$
\gamma: C \rightarrow M, \quad h \mapsto F_{h},
$$

where $F_{h}$ is obtained by substituting $\alpha_{i}$ for $a_{i}$ in $h$. When $h$ is viewed as a vector of length $p+1$ this gives the $p+1$ entries of a holomorphic vector-valued modular form $F_{h}$ of weight $(p, q+3 d)$. In particular, we have

$$
c(h)=\gamma(h) / \chi_{5}^{d}
$$

and we see that the order of $c(h)$ along $\mathfrak{H}_{1}^{2}$ is $\geq-d$. Using the expansion of $\chi_{6,3}$ and $\chi_{5}$ along $\mathfrak{H}_{1}^{2}$ given in Section 5 we can calculate the order $v\left(F_{h}\right)$ of $F_{h}$ along $\mathfrak{H}_{1}^{2}$. Division by $\chi_{5}^{v\left(F_{h}\right)}$ yields a holomorphic modular form on $\Gamma_{2}$ (with or without a character) of weight $\left(p, q+3 d-5 v\left(F_{h}\right)\right)$.

Conclusion 6.1 Every vector-valued modular form of given weight on $\Gamma_{2}$ can be constructed from a covariant by substituting the Fourier coefficients of $\chi_{6,3}$ and by multiplying with an appropriate power of $\chi_{5}$. The same holds for modular forms on $\Gamma_{2}$ with a character.

We shall see in the next sections that this gives a very effective way of constructing the Fourier expansions of vector-valued modular forms of degree 2. In fact, we can calculate the Fourier expansion of $\chi_{6,3}$ and $\chi_{5}$ easily as these are given in terms of theta functions.

Remark 6.2 The central role that $\chi_{6,3}$ plays can be explained as follows. It is wellknown that for a smooth curve $C$ of genus 2 there are six symmetric translates of the theta divisor, each isomorphic to $C$, in the $\operatorname{Jacobian} \operatorname{Jac}(C)$ passing through the origin. The tangents to these six curves give six points in the projectivized tangent space at the origin, that is, six points on $\mathbb{P}^{1}$. That is the way to retrieve the sextic as was remarked by Bolza, see [3, p. 481]. In terms of odd theta characteristics this means that if we write the Taylor expansion of the odd theta functions as

$$
\vartheta_{m_{i}}=c_{i, 1} z_{1}+c_{i, 2} z_{2}+\text { higher order terms, }
$$

then we get the form $\chi_{6,3}$ up to a normalization as the product of these linear terms

$$
\chi_{6,3} \sim \prod_{i=1}^{6}\left(c_{i, 1} z_{1}+c_{i, 2} z_{2}\right)=c_{1,1} \cdots c_{6,1} z_{1}^{6}+\cdots+c_{1,2} \cdots c_{6,2} z_{2}^{6}
$$




\section{Construction of modular forms}

In this section we give examples of constructions of vector-valued and scalar-valued modular forms using covariants. Our point of departure is the Fourier expansion of $\chi_{6,3}$ that we calculated as in (4) as a series in $Q_{1}$ and $Q_{2}$ up to $Q_{1}^{a} Q_{2}^{b}$ with $a+b \leq 170$.

$$
d=2
$$

We start with $d=2$, see Example 3.2. Covariants associated to the isotypical decomposition

$$
\operatorname{Sym}^{2}\left(\operatorname{Sym}^{6}(V)\right)=A[12,0]+A[10,2]+A[8,4]+A[6,6]
$$

provide by the construction given above modular forms in $S_{12,6}, S_{8,8}, S_{4,10}$ and $S_{0,12}$. Notice that all the latter spaces are one dimensional. The modular form in $S_{12,6}$ is the square of $\chi_{6,3}$ and this gives us its Fourier expansion immediately.

The covariant corresponding to $A[10,2]$ is (up to a scalar) the Hessian and we thus find a modular form $\chi_{8,8} \in S_{8,8}$ with coordinates (symmetric in the sense that interchanging $\alpha_{i}$ and $\alpha_{6-i}$ reverses the vector)

$$
\begin{array}{r}
\left(\alpha_{0} \alpha_{2}-\alpha_{1}^{2}, 4 \alpha_{0} \alpha_{3}-4 \alpha_{1} \alpha_{2}, 6 \alpha_{0} \alpha_{4}+4 \alpha_{1} \alpha_{3}-10 \alpha_{2}^{2}, 4 \alpha_{0} \alpha_{5}+16 \alpha_{1} \alpha 4-20 \alpha_{2} \alpha_{3},\right. \\
\left.\alpha_{0} \alpha_{6}+14 \alpha_{1} \alpha_{5}+5 \alpha_{2} \alpha_{4}-20 \alpha_{3}^{2}, \ldots, \alpha_{4} \alpha_{6}-\alpha_{5}^{2}\right)
\end{array}
$$

which we normalize such that its Fourier expansion starts with:

$$
\chi_{8,8}(\tau)=\left(\begin{array}{c}
0 \\
0 \\
\left(r-2+r^{-1}\right) \\
3\left(r-r^{-1}\right) \\
\left(4 r+10+4 r^{-1}\right) \\
3\left(r-r^{-1}\right) \\
\left(r-2+r^{-1}\right) \\
0 \\
0
\end{array}\right) q_{1} q_{2}+\ldots,
$$

where as before $q_{1}=e^{2 i \pi \tau_{1}}, q_{2}=e^{2 i \pi \tau_{2}}$ and $r=e^{2 i \pi \tau_{12}}$. By restriction along $\mathfrak{H}_{1} \times \mathfrak{H}_{1}$ we find a non-zero multiple of the transpose of $(0, \ldots, 0, \Delta \otimes \Delta, 0, \ldots, 0)$ which shows that $\chi_{8,8}$ is not divisible by $\chi_{5}$. Similarly, the covariant yielding a modular form in $S_{4,10}$ has coordinates that are reversed under interchanging $\alpha_{i}$ and $\alpha_{6-i}$

$$
\begin{gathered}
\left(\alpha_{0} \alpha_{4}-4 \alpha_{1} \alpha_{3}+3 \alpha_{2}^{2}, 2 \alpha_{0} \alpha_{5}-6 \alpha_{1} \alpha_{4}+4 \alpha_{2} \alpha_{3}, \alpha_{6} \alpha_{9}\right. \\
\left.-9 \alpha_{2} \alpha_{4}+8 \alpha_{3}^{2}, \ldots, \alpha_{2} \alpha_{6}-4 \alpha_{3} \alpha_{5}+3 \alpha_{4}^{2}\right) .
\end{gathered}
$$

We normalize the resulting form $\chi_{4,10}$ such that

$$
\chi_{4,10}(\tau)=\left(\begin{array}{c}
\left(r-2+r^{-1}\right) \\
2\left(r-r^{-1}\right) \\
3\left(r+6+r^{-1}\right) \\
2\left(r-r^{-1}\right) \\
\left(r-2+r^{-1}\right)
\end{array}\right) q_{1} q_{2}+\cdots
$$


Its restriction to $\mathfrak{H}_{1} \times \mathfrak{H}_{1}$ is a non-zero multiple of $(0,0, \Delta \times \Delta, 0,0)^{t}$, and we thus cannot divide by $\chi_{5}$. Finally, we get a non-zero form in $S_{0,12}$ by taking

$$
\chi_{12}=\alpha_{0} \alpha_{6}-6 \alpha_{1} \alpha_{5}+15 \alpha_{2} \alpha_{4}-10 \alpha_{3}^{2}
$$

which we normalize so that it starts with $\chi_{12}(\tau)=(r+10+1 / r) q_{1} q_{2}+\cdots$. One immediately gets the Fourier expansion of $\chi_{12}$.

In all these cases we checked the Fourier expansion by calculating Hecke eigenvalues for the Hecke operators $T(p)$ for primes $p \leq 23$ and verified that these fit with the values provided by [8] and [1]. We give the Hecke eigenvalues for $\chi_{8,8}$ and $\chi_{12,6}$ in a table in Section 10.

$$
d=3
$$

In this case we have the isotypical decomposition

$$
\begin{aligned}
\operatorname{Sym}^{3}\left(\operatorname{Sym}^{6}(V)\right)= & A[18,0]+A[16,2]+A[15,3]+A[14,4] \\
& +A[13,5]+2 A[12,6]+A[10,8]
\end{aligned}
$$

which by the procedure of the last section leads to modular forms of weights $(18,9),(14,11),(12,12),(10,13),(8,14),(6,15)$ and $(2,17)$ on the group $\Gamma_{2}$ with a character. If these forms are divisible by $\chi_{5}$ or $\chi_{10}$ we will find forms of smaller weight. Note that in the case of weight $(6,15)$ we find a 2-dimensional space of modular forms. In the cases of weight $(18,9),(14,11),(10,13),(2,17)$ the restriction of the corresponding form to $\mathfrak{H}_{1} \times \mathfrak{H}_{1}$ is a non-zero multiple of the transpose of a vector of the form

$$
(0, \ldots, 0, \Delta \delta \otimes \Delta \delta, 0, \ldots, 0)
$$

and thus gives non-zero modular forms and these forms are not divisible by $\chi_{5}$. We analyze the remaining three cases. In the case of weight $(12,12)$ our form vanishes with order 2 on $\mathfrak{H}_{1}^{2}$; dividing by $\chi_{10}$ yields a form $\chi_{12,2}$ on $\Gamma_{2}$ with character whose Fourier expansion starts with

$$
\chi_{12,2}(\tau)=\left(\begin{array}{c}
0 \\
0 \\
0 \\
2\left(R-R^{-1}\right) \\
9\left(R+R^{-1}\right) \\
12\left(R-R^{-1}\right) \\
0 \\
-12\left(R-R^{-1}\right) \\
-9\left(R+R^{-1}\right) \\
-2\left(R-R^{-1}\right) \\
0 \\
0 \\
0
\end{array}\right) Q_{1} Q_{2}+\cdots
$$


Note that its square $\operatorname{Sym}^{2} \chi_{12,2}$ is the generator of the space $S_{24,4}$. Similarly, in the case of weight $(8,14)$ our form vanishes with order 2 along $\mathfrak{H}_{1}^{2}$ and by dividing by $\chi_{10}$ we find a form of weight $(8,4)$ with character.

The representation $A[12,6]$ occurs with multiplicity 2 in $\operatorname{Sym}^{3}\left(\operatorname{Sym}^{6}(V)\right)$ and we thus can find two linearly independent covariants, say $h_{1}$ and $h_{2}$. The general linear combination of these two gives a modular form that upon restriction to $\mathfrak{H}_{1}^{2}$ yields a non-zero multiple of the transpose of

$$
\left[0,0,0, \eta^{12} \Delta\left(\tau_{1}\right) \otimes \eta^{12} \Delta\left(\tau_{2}\right), 0,0,0\right]
$$

The linear combination that vanishes along $\mathfrak{H}_{1}^{2}$ vanishes with multiplicity 2 and by division by $\chi_{10}$ we find the cusp form that generates the space of cusp forms of weight $(6,5)$ with character and its Fourier expansion starts with

$$
\chi_{6,5}(\tau)=\left(\begin{array}{c}
2\left(R-R^{-1}\right) \\
6\left(R+R^{-1}\right) \\
5\left(R-R^{-1}\right) \\
0 \\
5\left(R-R^{-1}\right) \\
6\left(R+R^{-1}\right) \\
2\left(R-R^{-1}\right)
\end{array}\right) Q_{1} Q_{2}+\cdots
$$

\section{Further examples}

Here we look at a few cases with $d=4$ and $d=5$.

We have the isotypical decomposition

$$
d=4
$$

$$
\begin{aligned}
\operatorname{Sym}^{4}\left(\operatorname{Sym}^{6}(V)\right)= & A[24,0]+A[22,2]+A[21,3] \\
& +2 A[20,4]+A[19,5]+3 A[18,6] \\
& +A[17,7]+3 A[16,8]+A[15,9] \\
& +2 A[14,10]+2 A[12,12] .
\end{aligned}
$$

We then use the covariants to construct modular forms from $\chi_{6,3}$. By calculating the behavior along $\mathfrak{H}_{1}^{2}$ we can deduce the orders of vanishing along $\mathfrak{H}_{1}^{2}$.

We claim that the following table gives the orders of the corresponding modular forms along $\mathfrak{H}_{1}^{2}$. If the multiplicity $\mu_{m, n}$ is greater than 1 we list the orders of vanishing occurring in the $\mu_{m, n}$-dimensional space of covariants.

From this table one can read off what modular forms can be constructed. For example, for the representation $A[18,6]$ (resp. $A[16,8]$ ) we find multiplicity 3 , hence a 3-dimensional space of cusp forms of weight $(12,18)$ (resp. weight $(8,20)$ ). We can calculate generating modular forms using the expressions for the covariants and observe that in both cases there is a 2-dimensional subspace of forms vanishing with order 2 along $\mathfrak{H}_{1}^{2}$. Dividing these forms by $\chi_{10}$ yields a 2 -dimensional space of cusp 


\begin{tabular}{rrrr}
\hline$[m, n]$ & $\mu_{m, n}$ & weight & order \\
\hline$[24,0]$ & 1 & $(24,12)$ & 0 \\
{$[22,2]$} & 1 & $(20,14)$ & 0 \\
{$[21,3]$} & 1 & $(18,15)$ & 2 \\
{$[20,4]$} & 2 & $(16,16)$ & 0,2 \\
{$[19,5]$} & 1 & $(14,17)$ & 2 \\
{$[18,6]$} & 3 & $(12,18)$ & $0,2,3$ \\
{$[17,7]$} & 1 & $(10,19)$ & 2 \\
{$[16,8]$} & 3 & $(8,20)$ & $0,2,3$ \\
{$[15,9]$} & 1 & $(6,21)$ & 2 \\
{$[14,10]$} & 2 & $(4,22)$ & 0,2 \\
{$[12,12]$} & 2 & $(0,24)$ & 2,4 \\
\hline
\end{tabular}

forms of weight $(12,8)$ (resp. $(8,10))$. By analyzing their behavior along $\mathfrak{H}_{1}^{2}$ we see that there is a 1 -dimensional subspace vanishing along $\mathfrak{H}_{1}^{2}$ and division by $\chi_{5}$ leads to a cusp form of weight $(12,3)$ (resp. $(8,5))$ with character. Alternatively, from the vanishing/non-vanishing of spaces of cusp forms (with/without a character) one can read off the orders of vanishing.

We give the details for $A[16,8]$. We find a 3-dimensional space of cusp forms $\gamma(h)$ in $M$ as $h$ ranges over the space of covariants associated to $d=4$ and $A[16,8]$. The generic element yields $\left[0, \ldots, 0, \Delta^{2} \otimes \Delta^{2}, 0, \ldots, 0\right]$ when restricted to $\mathfrak{H}_{1}^{2}$ and there is a 2-dimensional space of forms of weight $(8,10)$ vanishing at least doubly on $\mathfrak{H}_{1}^{2}$. Division by $\chi_{10}$ gives the 2-dimensional space of cusp forms of weight $(8,10)$ and it is generated by $G_{1}$ and $G_{2}$ with Fourier expansions

$$
G_{1}(\tau)=\left(\begin{array}{c}
\left(r-2+r^{-1}\right) \\
4\left(r-r^{-1}\right) \\
\left(9 r+34+9 r^{-1}\right) \\
13\left(r-r^{-1}\right) \\
15\left(r-2+r^{-1}\right) \\
13\left(r-r^{-1}\right) \\
\left(9 r+34+9 r^{-1}\right) \\
4\left(r-r^{-1}\right) \\
\left(r-2+r^{-1}\right)
\end{array}\right) q_{1} q_{2}+\cdots, \quad G_{2}(\tau)=\left(\begin{array}{c}
3\left(r-2+r^{-1}\right) \\
12\left(r-r^{-1}\right) \\
2\left(11 r+26+11 r^{-1}\right) \\
24\left(r-r^{-1}\right) \\
25\left(r-2+r^{-1}\right) \\
24\left(r-r^{-1}\right) \\
2\left(11 r+26+11 r^{-1}\right) \\
12\left(r-r^{-1}\right) \\
3\left(r-2+r^{-1}\right)
\end{array}\right) q_{1} q_{2}+\cdots
$$

The action of the Hecke operator $T_{2}$ on $G_{1}$ and $G_{2}$ is as follows:

$$
\left.G_{1}\right|_{8,10} T_{2}=8160 G_{1}-4080 G_{2},\left.\quad G_{2}\right|_{8,10} T_{2}=-2880 G_{1}-720 G_{2} .
$$

We get two Hecke eigenforms in $S_{8,10}$, namely $\chi_{8,10}^{( \pm)}=(37 \pm \sqrt{2185}) G_{1}-34 G_{2}$, on which the Hecke operator $T_{2}$ acts with eigenvalues $(3720 \pm 120 \sqrt{2185})$.

For the case of $A[18,6]$, leading to modular forms of weight $(12,18)$ and after division by $\chi_{10}$ to the 2 -dimensional space of cusp forms of weight $(12,8)$, the story is very similar and we can calculate the Fourier expansions and Hecke eigenvalues as well. We list these eigenvalues for weight $(8,10)$ and $(12,8)$ in the following table. 


\begin{tabular}{lrr}
\hline$q$ & $\lambda_{q}\left(\chi_{8,10}^{ \pm}\right)$ & $\lambda_{q}\left(\chi_{12,8}^{ \pm}\right)$ \\
\hline 2 & $3720 \pm 120 \sqrt{2185}$ & $-768 \pm 192 \sqrt{1381}$ \\
3 & $674280 \mp 18720 \sqrt{2185}$ & $86616 \pm 20736 \sqrt{1381}$ \\
4 & $-945536 \mp 28800 \sqrt{2185}$ & $790528 \mp 147456 \sqrt{1381}$ \\
5 & $-70706100 \pm 8188800 \sqrt{2185}$ & $362491500 \mp 5145600 \sqrt{1381}$ \\
7 & $-11441284400 \mp 446644800 \sqrt{2185}$ & $14252364592 \mp 459468288 \sqrt{1381}$ \\
\hline
\end{tabular}

The restriction of $G_{1}$ (resp. $G_{2}$ ) to $\mathfrak{H}_{1}^{2}$ is 52 (resp. 96) times the transpose of

$$
\left[0,0, e_{4} \Delta \otimes \Delta, 0,0,0, \Delta \otimes e_{4} \Delta, 0,0\right]
$$

so $G_{1} / 52-G_{2} / 96$ vanishes on $\mathfrak{H}_{1}^{2}$ and dividing it by $\chi_{5}$ gives the unique cusp form of weight $(8,5)$ on $\Gamma_{2}[2]$ which is $\mathfrak{S}_{6}$-anti-invariant.

Finally, in the case of $A[12,12]$ we find a 2-dimensional space of cusp forms of weight $(0,24)$. Every modular form in this space restricts to a multiple of $\Delta^{2} \otimes \Delta^{2}$ and there is a linear combination that vanishes on $\mathfrak{H}_{1}^{2}$ and it is a multiple of $E_{4} \chi_{10}^{2}$. Thus we see that by dividing by $\chi_{10}^{2}$ we get the Eisenstein series of weight 4 .

$$
d=5
$$

We list the irreducible representations occurring in the isotypical decomposition for $d=5$ in a table together with their multiplicities, the weight of the corresponding modular forms and the orders of vanishing along $\mathfrak{H}_{1}^{2}$.

\begin{tabular}{lrrrrrrr}
\hline$[m, n]$ & $\mu_{m, n}$ & weight & order & {$[m, n]$} & $\mu_{m, n}$ & weight & order \\
\hline$[30,0]$ & 1 & $(30,15)$ & 0 & {$[22,8]$} & 4 & $(14,23)$ & $0,2,3,4$ \\
{$[28,2]$} & 1 & $(26,17)$ & 0 & {$[21,9]$} & 3 & $(12,24)$ & $2,3,4$ \\
{$[27,3]$} & 1 & $(24,18)$ & 2 & {$[20,10]$} & 4 & $(10,25)$ & $0,2,3,4$ \\
{$[26,4]$} & 2 & $(22,19)$ & 0,2 & {$[19,11]$} & 2 & $(8,26)$ & 2,3 \\
{$[25,5]$} & 2 & $(20,20)$ & 2,3 & {$[18,12]$} & 4 & $(6,27)$ & $0,2,3,4$ \\
{$[24,6]$} & 3 & $(18,21)$ & $0,2,3$ & {$[17,13]$} & 1 & $(4,28)$ & 2 \\
{$[23,7]$} & 2 & $(16,22)$ & 2,3 & {$[16,14]$} & 2 & $(2,29)$ & 0,4 \\
\hline
\end{tabular}

We give the details for the case of $\lambda=(18,12)$, where the 4-dimensional space of covariants produces modular forms of weight $(6,27)$ with character. We find a 2 dimensional subspace of modular forms vanishing with multiplicity $\geq 3$, and dividing these by $\chi_{5}^{3}$ produces a basis of $S_{6,12}$ which is of dimension 2 . The two basis elements $G_{1}$ and $G_{2}$ have Fourier expansions starting with

$$
G_{1}(\tau)=\left(\begin{array}{c}
2\left(r+10+r^{-1}\right) \\
6\left(r-r^{-1}\right) \\
33\left(r-2+r^{-1}\right) \\
56\left(r-r^{-1}\right) \\
33\left(r-2+r^{-1}\right) \\
6\left(r-r^{-1}\right) \\
2\left(r+10+r^{-1}\right)
\end{array}\right) q_{1} q_{2}+\cdots, \quad G_{2}(\tau)=\left(\begin{array}{c}
0 \\
0 \\
\left(r-2+r^{-1}\right) \\
2\left(r-r^{-1}\right) \\
\left(r-2+r^{-1}\right) \\
0 \\
0
\end{array}\right) q_{1} q_{2}+\cdots .
$$


One calculates the action of the Hecke operator $T_{2}$ and finds

$$
\left.G_{1}\right|_{6,12} T_{2}=-2592 G_{1}+254016 G_{2},\left.\quad G_{2}\right|_{6,12} T_{2}=-480 G_{1}+24960 G_{2}
$$

and obtains eigenforms $\chi_{6,12}^{( \pm)}=(-41 \pm \sqrt{601}) G_{1}+756 G_{2}$.

In a similar way one finds a basis for the 2-dimensional spaces of cusp forms $S_{14,8}, S_{12,9}$ and $S_{10,10}$. One thus can calculate Hecke eigenvalues from the Fourier expansions. We give the results in the tables in Sect. 10 .

The cusp form $G_{2}$ vanishes identically along $\mathfrak{H}_{1}^{2}$ and $G_{2} / \chi_{5}$ generates the 1dimensional space $S_{6,7}\left(\Gamma_{2}, \epsilon\right)$.

\section{A final example: the space $S_{4,16}$}

In this section we illustrate the effectivity of our approach and show how one can use covariants to construct a basis for the 3-dimensional space $S_{4,16}$ and use this to calculate eigenvalues for the Hecke operators.

In the decomposition of $\operatorname{Sym}^{8}\left(\operatorname{Sym}^{6}(V)\right)$ the irreducible representation $A[26,22]$ of GL(2, $\mathbb{C})$ occurs with multiplicity 7 . By the construction of Section 6 this leads to a 7-dimensional subspace of $S_{4,46}$ vanishing with multiplicity $\geq 8$ at the divisor at infinity. All restrictions to $\mathfrak{H}_{1}^{2}$ of the cusp forms associated to the corresponding covariants are multiples of the transpose of $\left[0,0, \Delta^{4} \otimes \Delta^{4}, 0,0\right]$. In fact, we find a 6-dimensional subspace of forms vanishing with order $\geq 2$ along $\mathfrak{H}_{1}^{2}$. Dividing these forms by $\chi_{10}$ leads to a 6 -dimensional space of cusp forms of weight $(4,36)$. These forms restrict to multiples of the transpose of $\left[e_{4} \Delta^{3} \otimes \Delta^{3}, 0,0,0, \Delta^{3} \otimes e_{4} \Delta^{3}\right]$. Thus there is a 5-dimensional subspace of forms of weight $(4,36)$ vanishing along $\mathfrak{H}_{1}^{2}$ and these all vanish with multiplicity $\geq 2$ along $\mathfrak{H}_{1}^{2}$. We divide again by $\chi_{10}$ to get a 5 dimensional space of cusp forms of weight $(4,26)$. All the forms in this space restrict to multiples of the transpose of $\left[0,0, e_{4} \Delta^{2} \otimes e_{4} \Delta^{2}, 0,0\right]$ along $\mathfrak{H}_{1}^{2}$. This leads to a 4-dimensional space of cusp forms vanishing with multiplicity $\geq 1$ along $\mathfrak{H}_{1}^{2}$ and we divide these by $\chi_{5}$ to find a 4-dimensional space of cusp forms of weight $(4,21)$ with character. Since all these forms restrict to a multiple of the transpose of $\left[0, e_{6} \delta \Delta \otimes\right.$ $\left.e_{4} \delta \Delta, 0, e_{4} \delta \Delta \otimes e_{6} \delta \Delta, 0\right]$ it contains a 3 -dimensional subspace of cusp forms vanishing along $\mathfrak{H}_{1}^{2}$. Division by $\chi_{5}$ leads to the 3 -dimensional space of cusp forms of weight $(4,16)$. We normalize these forms such that their Fourier expansion starts with

$$
\begin{aligned}
& E_{1}(\tau)=\left(\begin{array}{c}
\left(r+10+r^{-1}\right) \\
2\left(r-r^{-1}\right) \\
3\left(r-2+r^{-1}\right) \\
2\left(r-r^{-1}\right) \\
\left(r+10+r^{-1}\right)
\end{array}\right) q_{1} q_{2}+\left(\begin{array}{c}
2\left(7 r^{2}+308 r+2106+308 r^{-1}+7 r^{-2}\right) \\
4\left(7 r^{2}+214 r-214 r^{-1}-7 r^{-2}\right) \\
2\left(27 r^{2}+504 r-1062+504 r^{-1}+27 r^{-2}\right) \\
4\left(10 r^{2}+76 r-76 r^{-1}-10 r^{-2}\right) \\
2\left(5 r^{2}+76 r+1134+76 r^{-1}+5 r^{-2}\right)
\end{array}\right) q_{1}^{2} q_{2}+\cdots \\
& E_{2}(\tau)=\left(\begin{array}{c}
\left(r+10+r^{-1}\right) \\
2\left(r-r^{-1}\right) \\
3\left(r-14+r^{-1}\right) \\
2\left(r-r^{-1}\right) \\
\left(r+10+r^{-1}\right)
\end{array}\right) q_{1} q_{2}+\left(\begin{array}{c}
2\left(-11 r^{2}+596 r+1566+596 r^{-1}-11 r^{-2}\right) \\
4\left(11 r^{2}+1006 r-1006 r^{-1}-11 r^{-2}\right) \\
2\left(9 r^{2}+2088 r+5310+2008 r^{-1}+9 r^{-2}\right) \\
4\left(10 r^{2}+76 r-76 r^{-1}-10 r^{-2}\right) \\
2\left(5 r^{2}+76 r+1134+76 r^{-1}+5 r^{-2}\right)
\end{array}\right) q_{1}^{2} q_{2}+\cdots
\end{aligned}
$$




$$
\begin{aligned}
E_{3}(\tau)= & \left(\begin{array}{c}
\left(5 r+104+5 r^{-1}\right) \\
10\left(r-r^{-1}\right) \\
\left(15 r-138+15 r^{-1}\right) \\
10\left(r-r^{-1}\right) \\
\left(5 r+104+5 r^{-1}\right)
\end{array}\right) q_{1} q_{2} \\
+ & \left(\begin{array}{c}
2\left(35 r^{2}+3808 r+18306+3808 r^{-1}+35 r^{-2}\right) \\
4\left(35 r^{2}+3338 r-3338 r^{-1}-35 r^{-2}\right) \\
2\left(243 r^{2}+8568 r+10890+8568 r^{-1}+243 r^{-2}\right) \\
4\left(104 r^{2}+1892 r-1892 r^{-1}-104 r^{-2}\right) \\
8\left(13 r^{2}+473 r+2106+473 r^{-1}+13 r^{-2}\right)
\end{array}\right) q_{1}^{2} q_{2}+\cdots
\end{aligned}
$$

The restrictions of the $E_{i}$ along the diagonal are the transposes of $12\left[e_{4}^{2} \Delta \otimes\right.$ $\left.e_{4} \Delta, 0,0,0, e_{4} \Delta \otimes e_{4}^{2} \Delta\right], 12\left[e_{4}^{2} \Delta \otimes e_{4} \Delta, 0,-3 e_{6} \Delta \otimes e_{6} \Delta, 0, e_{4}^{2} \Delta \otimes e_{4} \Delta\right]$ and $6\left[19 e_{4}^{2} \Delta \otimes e_{4} \Delta, 0,-18 e_{6} \Delta \otimes e_{6} \Delta, 0,19 e_{4}^{2} \Delta \otimes e_{4} \Delta\right]$. So the orders of vanishing along $\mathfrak{H}_{1}^{2}$ in the 7 -dimensional space of cusp forms of weight $(4,46)$ that is the image under the map $\gamma$ of the covariants given by $A[26,22]$ are $\{0,2,4,5,6,7\}$. Finally we remark that the form $\left(E_{1} / 12+E_{2} / 26-E_{3} / 78\right) / \chi_{5}$ gives a cusp form that generates the space of cusp forms of weight $(6,11)$ with character.

Using the Fourier expansions that we got we can calculate Hecke eigenvalues. Let $\alpha$ be a root of the irreducible polynomial $P=x^{3}-1042 x^{2}+215915 x+6800500$ in $\mathbb{Q}[x]$. This field has discriminant 43803704 and a basis of the ring of integers of $K=\mathbb{Q}(\alpha)$ is given by $1, \alpha$ and $\beta=(1 / 16785) \alpha^{2}+(1062 / 1865) \alpha+3007 / 3357$. Then a Hecke eigenform $\chi_{4,16}=\chi_{4,16}^{(\alpha)}$ with integral Fourier coefficients is given by

$$
\begin{aligned}
\chi_{4,16}= & (405064 \alpha-662955 \beta+1366955) E_{1}+435 \alpha E_{2} \\
& +(-61595 \alpha+100650 \beta-210400) E_{3} .
\end{aligned}
$$

The Hecke eigenvalues generate the totally real field $\mathbb{Q}(\alpha)$ of degree 3 over $\mathbb{Q}$ of discriminant 43803704. We give some Hecke eigenvalues for this form.

\begin{tabular}{lr}
\hline$q$ & $\lambda_{q}\left(\chi_{4,16}\right)$ \\
\hline 2 & $192 \alpha$ \\
3 & $157261824 \alpha-230584320 \beta-11214073856$ \\
4 & $-15895326720 \alpha+27887846400 \beta-41100006690$ \\
5 & $-7428303065088 \alpha+12024244661760 \beta+44274749992240$ \\
7 & $160404429828096 \alpha-252069882854400 \beta-377719068915351$ \\
9 &
\end{tabular}

Harder predicted in [11] congruences between elliptic modular forms and Siegel modular forms. In the case at hand, the critical value at $s=20$ of the $L$-series $\Gamma(s)(2 \pi)^{-s} L(f, s)$ of a Hecke eigenform $f=\sum_{n} a(n) q^{n}$ of weight 34 for $\operatorname{SL}(2, \mathbb{Z})$ is divisible by the prime 1571 and therefore we expect for every prime $p$ a congruence of the form 


$$
\lambda_{p}\left(\chi_{4,16}\right) \equiv p^{14}+a(p)+p^{19} \bmod \ell
$$

where $\ell$ is a prime dividing 1571 in the composite of the fields $\mathbb{Q}(\alpha)$ and $\mathbb{Q}(\sqrt{2356201})$ of eigenvalues $\lambda_{p}\left(\chi_{4}, 16\right)$ of $\chi_{4,16}$ and $a(p)$ of $f$ for appropriate choices of the eigenforms $\chi_{4,16}^{(\alpha)}$ and $f$. One checks that the norm of $\lambda_{p}\left(\chi_{4,16}\right)-\left(p^{14}+a(p)+p^{19}\right)$ is indeed divisible by 1571 for $p=2,3,5$ and 7 . More precisely, we let $f_{a} \in S_{34}\left(\Gamma_{1}\right)$ be the form

$$
f_{a}=q+a q^{2}+(-22140-312 a) q^{3}+\cdots
$$

with $a$ being a root of $Q=x^{2}+121680 x-8513040384$. The minimal polynomials $P$ and $Q$ of $a$ and $\alpha$ split in $\mathbb{F}_{1571}[x]$ as $(x-851)(x-7)$ and $(x-63)(x-1146)(x-1404)$. One sees that the congruence is satisfied for the modular forms $f_{a}$ and $\chi_{4,16}=\chi_{4,16}^{(\alpha)}$ for $p=2,3,5$ and 7 for the pair $(a, \alpha)$ corresponding to $(851,63) \bmod 1571$ only.

\section{Tables}

In this section we give the Hecke eigenvalues of some modular forms constructed above using covariants. We checked that these eigenvalues are consistent with the results of $[1,2,8]$. The form $\chi_{8,8}$ generates $S_{8,8}$ and $\chi_{12,6}$ generates $S_{12,6}$. The forms $\chi_{6,12}^{+}, \chi_{6,12}^{-}$form a basis of the 2-dimensional space $S_{6,12}$; similar notation is used for weights $(10,10),(12,9)$ and $(14,8)$.

\begin{tabular}{rrr}
\hline$q$ & $\lambda_{q}\left(\chi_{8,8}\right)$ & $\lambda_{q}\left(\chi_{12,6}\right)$ \\
\hline 2 & 1344 & -240 \\
3 & -6408 & 68040 \\
4 & 28672 & 1118464 \\
5 & -30774900 & 1476510 \\
7 & 451366384 & -334972400 \\
9 & -3092097159 & -5279708871 \\
11 & 13030789224 & 3580209624 \\
13 & -328006712228 & 91151149180 \\
17 & 5520456217764 & -11025016477020 \\
19 & -28220918878760 & -22060913325080 \\
23 & 79689608755152 & 195863810691120 \\
\hline
\end{tabular}

\begin{tabular}{lrr}
\hline$q$ & $\lambda_{q}\left(\chi_{6,12}^{ \pm}\right)$ & $\lambda_{q}\left(\chi_{10,10}^{ \pm}\right)$ \\
\hline 2 & $11184 \pm 336 \sqrt{601}$ & $11400 \pm 120 \sqrt{11041}$ \\
3 & $-167832 \mp 157248 \sqrt{601}$ & $480600 \pm 4320 \sqrt{11041}$ \\
4 & $-26121728 \pm 5451264 \sqrt{601}$ & $149854336 \pm 339840 \sqrt{11041}$ \\
5 & $-554158500 \pm 77280000 \sqrt{601}$ & $-1325429700 \mp 25276800 \sqrt{11041}$ \\
7 & $-28518281456 \pm 177641856 \sqrt{601}$ & $236903926000 \mp 658324800 \sqrt{11041}$ \\
\hline
\end{tabular}




\begin{tabular}{lrr}
\hline$q$ & $\lambda_{q}\left(\chi_{12,9}^{ \pm}\right)$ & $\lambda_{q}\left(\chi_{14,8}^{ \pm}\right)$ \\
\hline 2 & $-6216 \pm 72 \sqrt{25249}$ & $-2016 \pm 96 \sqrt{9961}$ \\
3 & $1074168 \mp 16416 \sqrt{25249}$ & $2762568 \pm 18432 \sqrt{9961}$ \\
4 & $-40492928 \mp 784512 \sqrt{25249}$ & $-80611328 \pm 1050624 \sqrt{9961}$ \\
5 & $-1795354500 \pm 3600000 \sqrt{25249}$ & $-951372900 \mp 12134400 \sqrt{9961}$ \\
7 & $-147859080656 \mp 507187008 \sqrt{25249}$ & $87767118544 \mp 804225024 \sqrt{9961}$ \\
\hline
\end{tabular}

Using these tables one can check Harder's conjecture [11] on the existence of congruences between Siegel modular forms and elliptic modular forms. In [9, p. 240] one finds for the cases of weights $(6,12),(10,10),(12,9)$ and $(14,8)$ predicted congruences with the eigenform $f_{28}^{ \pm} \in S_{28}\left(\Gamma_{1}\right)$ modulo 823, 157, 4057 and 647. The congruence for the eigenvalues for $T_{2}$ was checked in [9, p. 239] and one can verify that these congruences hold for the eigenvalues of $T_{3}, T_{5}$ and $T_{7}$ as well. For example for $(j, k)=(12,9)$, Harder predicts for every prime $p$ a congruence

$$
\lambda(p) \equiv p^{k-2}+a(p)+p^{j+k-1} \bmod \ell
$$

for the Hecke eigenvalues $\lambda(p)$ and $a(p)$ of a pair $(F, f)$ with $F$ equal to an eigenform in $S_{12,9}\left(\Gamma_{2}\right)$ and $f$ an eigenform in $S_{28}\left(\Gamma_{1}\right)$, where $\ell$ is a prime dividing 4057 in the composite of the fields of eigenvalues of $f_{28}^{ \pm}$and $\chi_{12,9}^{ \pm}$. One checks that the norm of

$$
\begin{aligned}
& 147859080656+507187008 \sqrt{25249}+7^{7} \\
& -87695981800-809077248 \sqrt{18209}+7^{20}
\end{aligned}
$$

in $\mathbb{Q}(\sqrt{25249}, \sqrt{18209})$ is divisible by 4057 .

Acknowledgements The first author thanks the Max-Planck-Institut für Mathematik for the hospitality he is enjoying there and the third author thanks YMSC of Tsinghua University for its hospitality.

Open Access This article is distributed under the terms of the Creative Commons Attribution 4.0 International License (http://creativecommons.org/licenses/by/4.0/), which permits unrestricted use, distribution, and reproduction in any medium, provided you give appropriate credit to the original author(s) and the source, provide a link to the Creative Commons license, and indicate if changes were made.

\section{References}

1. Bergström, J., Faber, C., van der Geer, G.: Siegel modular forms of genus 2 and level 2: cohomological computations and conjectures. Int. Math. Res. Not. IMRN 2008, Art. ID rnn100, 20 p (2008)

2. Bergström, J., Faber, C., van der Geer, G.: Siegel modular forms of degree three and the cohomology of local systems. Selecta Math. (N.S.) 20(1), 83-124 (2014)

3. Bolza, O.: Darstellung der rationalen ganzen Invarianten der Binärform sechsten Grades durch die Nullwerte der zugehörigen $\theta$-Functionen. Math. Ann. 30, 478-495 (1887)

4. Chipalkatti, J.: Decomposable ternary cubics. Experiment. Math. 11, 69-80 (2002)

5. Clebsch, A.: Theorie der binären algebraischen Formen. Leipzig (1872)

6. Cléry, F., van der Geer, G.: Constructing vector-valued Siegel modular forms from scalar-valued Siegel modular forms. Pure and Applied Mathematics Quarterly 11(1), 21-47 (2015)

7. Cléry, F., van der Geer, G., Grushevsky, S.: Siegel modular forms of genus 2 and level 2. Int. J. Math. 26(05), 1550034, 51 p (2015) 
8. Faber, C., van der Geer, G.: Sur la cohomologie des systèmes locaux sur les espaces des modules des courbes de genre 2 et des surfaces abéliennes, I, II. C.R. Acad. Sci. Paris, Sér. I338, 381-384, 467-470 (2004)

9. van der Geer, G.: Siegel modular forms and their applications. In: J. Bruinier, G. van der Geer, G. Harder, D. Zagier: The 1-2-3 of modular forms. Universitext. Springer Verlag (2007)

10. Grace, J.H., Young, A.: The algebra of invariants. Cambridge University Press, Cambridge (1903)

11. Harder, G.: A congruence between a Siegel and an elliptic modular form. In: J. Bruinier, G. van der Geer, G. Harder, D. Zagier: The 1-2-3 of modular forms. Universitext. Springer Verlag (2007)

12. Ibukiyama, T.: An answer to van der Geer's question: construction of the vector valued Siegel cusp form of $\operatorname{Sp}(2, \mathbb{Z})$ of weight $\operatorname{det}^{8} \operatorname{Sym}^{6}$. Unpublished manuscript (2001)

13. Igusa, J.-I.: Arithmetic moduli for genus two. Annals of Math. 72, 612-649 (1960)

14. Igusa, J.-I.: On Siegel modular forms of genus two. Amer. J. of Math. 84, 175-200 (1962)

15. Igusa, J.-I.: On Siegel modular forms of genus two (II). Amer. J. of Math. 86, 392-412 (1964)

16. Igusa, J.-I.: Modular forms and projective invariants. Amer. J. Math. 89, 817-855 (1967)

17. Igusa, J.-I.: On the ring of modular forms of degree two over $\mathbb{Z}$. Amer. J. Math. 101, 149-183 (1979)

18. Kaneko, M., Zagier, D.: A generalized Jacobi theta function and quasimodular forms. In: The Moduli Space of Curves (R. Dijkgraaf, C. Faber, G. van der Geer, eds.), Prog. in Math. 129, Birkhäuser, Boston, pp. 165-172 (1995)

19. Klingen, H.: Zur Struktur der Siegelschen Modulgruppe. Math. Z. 136, 169-178 (1974)

20. Maass, H.: Die Multiplikatorsysteme zur Siegelschen Modulgruppe. Nachr. Akad. Wiss. Göttingen Math.-Phys. Kl. II, pp. 125-135 (1964)

21. Mumford, D., Fogarty, J.: Geometric invariant theory. Ergebnisse der Mathematik und ihrer Grenzgebiete 34. 2nd edition, (1981)

22. Mumford, D., Suominen, K.: Introduction to the Theory of Moduli. In: Algebraic geometry, Oslo 1970 (Proc. Fifth Nordic Summer-School in Math.), pp. 171-222. Wolters-Noordhoff, Groningen, (1972)

23. Springer, T.A.: Invariant Theory. Lecture Notes in Mathematics 585, Springer Verlag (1977)

24. Vistoli, A.: The Chow ring of $\mathcal{M}_{2}$. Appendix to a paper by Edidin and Graham. Invent. Math. 131, 635-644 (1998)

25. Zagier, D.: Elliptic modular forms and their applications. In: J. Bruinier, G. van der Geer, G. Harder, D. Zagier: The 1-2-3 of modular forms. Universitext. Springer Verlag (2007) 